\title{
Fungi found in Mediterranean and North Sea sponges: How specific are they?
}

\author{
Mohd Azrul Naim ${ }^{1,2}$, Hauke Smidt ${ }^{1}$, Detmer Sipkema ${ }^{\text {Corresp. } 1}$ \\ 1 Laboratory of Microbiology, Wageningen University, Wageningen, Netherlands \\ 2 Department of Biotechnology, International Islamic University, Jalan Istana, Malaysia \\ Corresponding Author: Detmer Sipkema \\ Email address: detmer.sipkema@wur.nl
}

Fungi and other eukaryotes represent one of the last frontiers of microbial diversity in the sponge holobiont. In this study we employed pyrosequencing of $18 \mathrm{~S}$ ribosomal RNA gene amplicons containing the $\mathrm{V} 7$ and $\mathrm{V} 8$ hypervariable regions to explore the fungal diversity of seven sponge species from the North Sea and the Mediterranean Sea. For most sponges, fungi were present at a low relative abundance averaging $0.75 \%$ of the $18 \mathrm{~S}$ rRNA gene reads. In total, 44 fungal OTUs (operational taxonomic units) were detected in sponges, and 28 of these OTUs were also found in seawater. Twenty-two of the spongeassociated OTUs were identified as yeasts (mainly Malasseziales), representing $84 \%$ of the fungal reads. Several OTUs were related to fungal sequences previously retrieved from other sponges, but all OTUs were also related to fungi from other biological sources, such as seawater, sediments, lakes and anaerobic digesters. Therefore our data, supported by currently available data, point into the direction of mostly accidental presence of fungi in sponges and do not support the existence of a sponge-specific fungal community. 
1 Fungi found in Mediterranean and North Sea Sponges: How specific

2 are they?

3

4

5

6

7

8

9

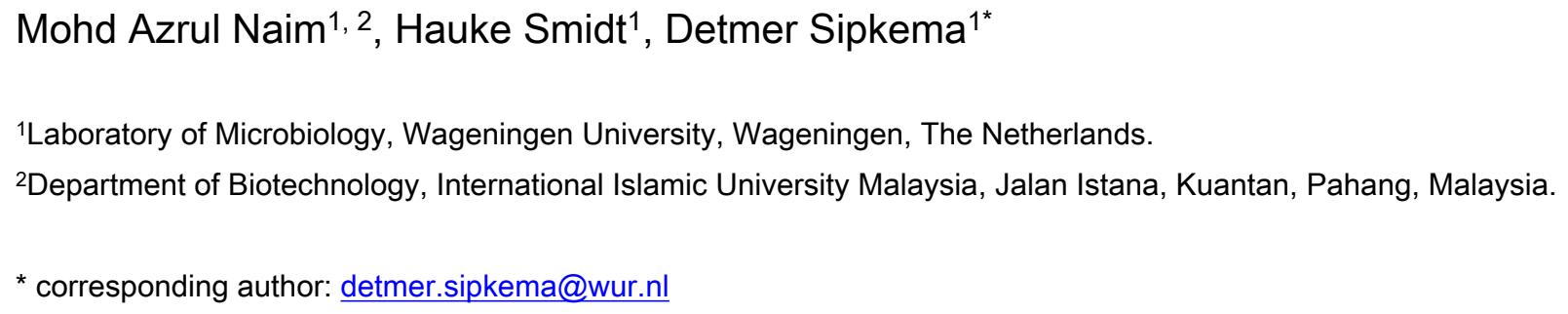

\section{Abstract}

Fungi and other eukaryotes represent one of the last frontiers of microbial diversity in the sponge holobiont. In this study we employed pyrosequencing of 18S ribosomal RNA gene amplicons containing the V7 and V8 hypervariable regions to explore the fungal diversity of seven sponge species from the North Sea and the Mediterranean Sea. For most sponges, fungi were present at a low relative abundance averaging $0.75 \%$ of the $18 \mathrm{~S}$ rRNA gene reads. In total, 44 fungal OTUs (operational taxonomic units) were detected in sponges, and 28 of these OTUs were also found in seawater. Twenty-two of the sponge-associated OTUs were identified as yeasts (mainly Malasseziales), representing $84 \%$ of the fungal reads. Several OTUs were related to fungal sequences previously retrieved from other sponges, but all OTUs were also related to fungi from other biological sources, such as seawater, sediments, lakes and anaerobic digesters. Therefore our data, supported by currently available data, point into the direction of mostly accidental presence of fungi in sponges and do not support the existence of a sponge-specific fungal community. 


\section{Introduction}

Fungi constitute a large proportion of microbial diversity on Earth (Hawksworth 2001; Mueller \& Schmit 2007) and are considered key players in terrestrial environments for decomposition of organic matter, nutrient recycling or as symbionts of plants or other fungi by improving host fitness (Rodriguez et al. 2004). Global fungal richness has been estimated between 1.5 to 1.6 million species (Hawksworth 1991; Hawksworth 2001), but despite extensive attempts to study and characterize fungi, their diversity remains underexplored. Most of our knowledge about evolution and ecology of fungi has been derived from cultured representatives of fungi from the terrestrial environment. In comparison, much less is known about marine fungal diversity and ecology.

Marine fungi belong to a wide variety of families, but appear to be present only in low numbers (compared to bacteria) in seawater and have been estimated to contribute up to only $0.6 \%$ of the global fungal richness (Richards et al. 2012a; Richards et al. 2015). The generally accepted definition of a marine fungus is broad and is based on the habitat as described by Kohlmeyer and Volkmann-Kohlmeyer: "obligate marine fungi are those that grow and sporulate exclusively in a marine or estuarine habitat; facultative marine fungi are those from freshwater and terrestrial milieus able to grow and possibly also sporulate in the marine environment" (Kohlmeyer \& Volkmann-Kohlmeyer 1990). Fungi are considered to play a role in marine ecosystems as saprotrophs, parasites, or symbionts (Hyde et al. 1998). Different habitats of marine fungi have been studied including deep-sea sediments (Singh et al. 2010), hydrothermal vents (Le Calvez et al. 2009), seawater (Kis-Papo et al. 2003) and anoxic regions in the deeper parts of the oceans (Bass et al. 2007). Marine fungi have also been described to be associated to marine animals, such as sea fans (Toledo-Hernández et al. 2008), corals (Amend et al. 2012; Bentis et al. 2000) and algae (Loque et al. 2009).

Marine sponges provide yet another habitat for fungi, but whereas bacterial and archaeal diversity in sponges has been thoroughly characterized (Simister et al. 2012; Taylor et al. 2007), knowledge of sponge-associated fungal diversity remains scarce (Webster \& Taylor 2012). Indirect evidence of interactions between marine sponges and fungi was provided by the detection of fungal introns in the genomes of some marine sponge species that were most probably acquired by horizontal gene transfer (Rot et al. 2006). Fungi have been repeatedly isolated from many sponge species (Baker et al. 2009; Höller et al. 2000; Liu et al. 2010; Passarini et al. 2013; Paz et al. 2010; Pivkin et al. ; Wang et al. 2007; Wiese et al. 2011). Despite a gap of knowledge about the fungal life cycle in sponges and other environmental fungi (Richards et al. 2012b), it is enticing to speculate about the role of sponge-associated fungi. Many sponge-derived fungi have been found to produce molecules with antimicrobial activity and may be involved in chemical protection of their hosts (Indraningrat et al. 2016). In addition, they are potentially parasites or pathogens to sponges themselves or sponges may solely serve as a reservoir for (pathogenic) marine fungi once they (or their spores) are trapped by the sponge through its efficient water filtration system. For instance, Metchniskowia spp., which were found in H. simulans and Antarctic sponges (Baker et al. 2009; Vaca et 
al. 2013), are known to be responsible for the infection and mortality of prawns (Chen et al. 2007) and Aspergillus sydowii, a pathogen of sea fans was also isolated from the marine sponge Spongia obscura (Ein-Gil et al. 2009). However, the true diversity of spongeassociated fungi has until recently been difficult to establish (Schippers et al. 2012). The reason is that designing specific PCR primers for universal fungal phylogenetic marker genes remains challenging since sponges and fungi are closely related from an evolutionary perspective (Borchiellini et al. 1998). This was demonstrated in a Hawaiian sponge study where many sponge-derived sequences were found in clone libraries generated from PCR amplicons using 'fungi-specific' 18S rRNA gene and ITS primers (Gao et al. 2008; Jin et al. 2014). With the use of next-generation sequencing, it is now possible to overcome such interference of the sponge host phylogenetic marker genes by the sheer number of reads that is generated as shown in a number of recent studies (He et al. 2014; Passarini et al. 2015; Rodríguez-Marconi et al. 2015; Wang et al. 2014). The aim of this study was to assess the diversity and specificity of sponge-associated fungi. Seven shallow water sponge species from two different regions, the North Sea and the Mediterranean Sea, were sampled to identify host specificity of the associated fungal communities and the impact of geography on community structuring.

\section{Materials and methods}

\section{Sample collection and processing}

The North Sea sponges Halichondria panicea (P1-P3), Haliclona xena (X1-X3), and Suberites massa (M1-M3) were collected on December $3^{\text {rd }}$, 2008, from the Oosterschelde estuary, at the dive site Lokkersnol (N 51 $\left.38^{\prime} 58.07^{\prime \prime}, 3^{\circ} 53^{\prime} 5.11^{\prime \prime}\right)$ by SCUBA diving at a depth of approximately $14 \mathrm{~m}$. Sponge collection of the North Sea sponges was approved by the Provincie Zeeland (document number 0501560). The Mediterranean sponges Aplysina aerophoba (A1-A3), Petrosia ficiformis (F1-F3), Axinella damicornis (D1-D3) and Axinella verrucosa (V1-V3) were collected by SCUBA diving offshore L'Escala, Spain (N 42 $06^{\prime} 52.20^{\prime \prime}, \mathrm{E} 03^{\circ} 10^{\prime} 06.52^{\prime \prime}$ ) at a depth of approximately $15 \mathrm{~m}$ on January $15^{\text {th }}, 2012$. The collection of Mediterranean sponge samples was conducted in strict accordance with Spanish and European regulations within the rules of the Spanish National Research Council with the approval of the Directorate of Research of the Spanish Government. Initial identification of sponges based on their morphology was done by Prof. Rene Wijfels and Dr. Klaske Schippers for the North Sea sponges and by Dr. Detmer Sipkema and Prof. M.J Uriz for the Mediterranean sponges. All sponge specimens from the North Sea and the Mediterranean Sea were collected in triplicate. Specimens were brought to the surface in ziplock plastic bags and were immediately transported to the laboratory in excess of seawater and processed. Each sponge specimen was separately submerged and cut into pieces of approximately $0.5 \mathrm{ml}$ that contained both pinacoderm and choanoderm and was rinsed three times in a large volume of autoclaved artificial seawater $\left(26.52 \mathrm{~g} \mathrm{NaCl}, 2.45 \mathrm{~g} \mathrm{MgCl}_{2}, 0.73 \mathrm{~g} \mathrm{KCl}, 1.14 \mathrm{~g}\right.$ $\mathrm{CaCl}_{2}$ and $3.31 \mathrm{~g} \mathrm{MgSO}_{4} / \mathrm{l}$ ) and kept at $-80^{\circ} \mathrm{C}$ until further processing. Furthermore, between 5 and 10 liters of seawater from both locations was collected and filtered 
112 immediately upon collection onto a $0.2 \mu \mathrm{m}$ polycarbonate filter with a diameter of $47 \mathrm{~mm}$ 113 (GE Osmonics, Minnetonka, MN, USA). Each filter was then stored in a sterile $15 \mathrm{ml}$ 114 Falcon tube and kept at $-80^{\circ} \mathrm{C}$ until further processing.

115

\section{DNA extraction and PCR amplification}

Total DNA was extracted from North Sea sponges using the DNeasy Blood \& Tissue Kit (Qiagen, Hilden, Germany) according to the tissue extraction protocol. For Mediterranean sponges total DNA was extracted using the FastDNA SPIN kit for soil (MP Biomedicals, Solon, OH, USA) with the aid of a PreCellys ${ }^{\circledR}$ homogenizer (Bertin Technologies, France) following the manufacturers' protocol. For seawater samples, filters were cut in two pieces, and DNA was extracted from half of the filter with the FastDNA SPIN kit for soil following the same protocol that was used for the Mediterranean sponges.

Amplification of partial 18S rRNA genes was performed using the GoTaq ${ }^{\circledR}$ Hot Start Polymerase kit (Promega $\mathrm{GmbH}$, Mannheim, Germany) with the universal fungal primers FF390 (CGATAACGAACGAGACCT) and FR1 (ANCCATTCAATCGGTANT) (Vainio \& Hantula 2000), which amplify a region of approximately 350 base pairs that includes the V7 and V8 hypervariable regions of the eukaryotic small-subunit rRNA gene. Samplespecific barcodes and adapter sequences were added to the forward primer as described previously (Hamady et al. 2008) (Table S1). The PCR conditions were: initial denaturation $\left(2 \mathrm{~min}\right.$ at $95^{\circ} \mathrm{C}$ ) followed by 30 cycles of denaturation $\left(30 \mathrm{~s}\right.$ at $\left.95^{\circ} \mathrm{C}\right)$, primer annealing $\left(45 \mathrm{~s}\right.$ at $\left.50{ }^{\circ} \mathrm{C}\right)$, primer extension $\left(60 \mathrm{~s}\right.$ at $\left.72{ }^{\circ} \mathrm{C}\right)$, and a final extension $\left(10 \mathrm{~min}\right.$ at $\left.72{ }^{\circ} \mathrm{C}\right)$. The final PCR mixture $(50 \mu \mathrm{l})$ contained $1 \times \mathrm{GoTaq}^{\circledR}$ Green Flexi buffer, $1.5 \mathrm{mM} \mathrm{MgCl}$, $0.2 \mathrm{mM}$ of each dNTP, $0.2 \mu \mathrm{M}$ of each primer, $1.25 \mathrm{U} \mathrm{GoTaq}^{\circledR}$ Hot Start Polymerase and $10 \mathrm{ng}$ template DNA. PCR reactions were carried out in triplicate, pooled and cleaned using the High Pure PCR Cleanup Micro Kit (Roche Diagnostics $\mathrm{GmbH}$, Mannheim, Germany). Purified DNA concentrations were measured with a Qubit ${ }^{\circledR} 2.0$ Fluorometer (Invitrogen, Carlsbad, CA, USA). An equimolar mixture with a final concentration of 1 $\mu \mathrm{g} / \mathrm{ml}$ PCR product was prepared, electrophoresed on $1.25 \%(\mathrm{w} / \mathrm{v})$ agarose gel and subsequently purified using the Milipore DNA Gel Extraction Kit (Milipore, Billerica, MA, USA). The pooled purified DNA was pyrosequenced on a 454 Roche platform at GATC Biotech, Konstanz, Germany. Pyrosequencing data was deposited at the European Bioinformatics Institute with accession numbers ERS225550 - ERS225575.

\section{Sequence analysis}

Pyrosequencing data was analysed using the QIIME pipeline v1.5.0 (Caporaso et al. 2010b). Low quality sequences were removed using default parameters, including (i) reads with fewer than 200 or more than 1000 nucleotides; (ii) reads with more than six ambiguous nucleotides, (iii) homopolymer runs exceeding six nucleotides, (iv) reads with missing quality scores and reads with a mean quality score lower than 25 , and (v) reads with mismatches in the primer sequence. Operational taxonomic units (OTUs) were identified at the 97\% identity level using UCLUST v1.2.22 embedded in QIIME (Edgar 2010). Representative sequences from the OTUs were aligned using PyNAST (Caporaso 
et al. 2010a) against the aligned SILVA 104 core set. Taxonomic assignment of all OTUs was performed using the BLAST algorithm against the QIIME-compatible version of the SILVA 104 release (Pruesse et al. 2007) as reference database. Possible chimeric OTUs were identified using QIIME's ChimeraSlayer and removed from the initially generated OTU list, producing a final set of 585 non-chimeric OTUs.

Since the majority of reads and OTUs were not of fungal origin, these were removed from the dataset prior to further analysis. The fungal OTU matrix from sponge and seawater samples was used to calculate species richness estimates using the 'Species Frequency/Abundance Data' option with default settings with the SPADE program (Chao \& Shen 2013). Fungal community coverage was estimated using Good's coverage in which Coverage $=1$ - (number of singleton OTUs / number of reads). In addition, the fungal OTU matrix was used for betadiversity analysis and samples that had $<10$ fungal reads were excluded from this analysis. Bray Curtis dissimilarity was calculated based on square root transformed relative abundance data and on presence-absence. Principal coordinates analysis (PCoA) was performed to represent the samples in a low dimensional space. All statistical analyses were performed using the multivariate statistical software package Primer V7 (Primer-E Ltd, Plymouth, UK).

\section{Phylogenetic analysis of sponge-associated fungi}

For a more detailed phylogenetic analysis of the fungal OTUs from sponges we selected all fungal OTUs that were found in sponge samples excluding only singletons. Representative reads for these OTUs were deposited at NCBI genbank with accession numbers MF094397-MF094440. These representative sequences were aligned using the SILVA online SINA alignment service (Pruesse et al. 2007). Each OTU was complemented with the two most closely related 18S rRNA gene sequences as determined by a BLAST search against the NCBI nucleotide database. Nearest neighbour sequences and published 18S rRNA sponge-derived fungal sequences longer than 700 nucleotides (Baker et al. 2009; Simister et al. 2012) were downloaded from the SILVA database (release 108) and together with aligned OTUs from our own dataset imported into the ARB software package (Ludwig et al. 2004). Nearest neighbour sequences and published sponge-derived fungal 18S rRNA sequences were first used to construct a Bayesian phylogenetic tree. Ambiguous regions of the alignment were systematically removed using the program Gblocks v.0.91b (Castresana 2000). The default program parameters were used, except allowing a minimum block length of three and gaps in $50 \%$ of positions. Phylogenetic trees were created by Bayesian analysis, using MrBayes v3.2 (Ronquist et al. 2012) at the freely available Bioportal server (www.bioportal.uio.no). All parameters were treated as unknown variables with uniform prior-probability densities at the beginning of each run, and their values were estimated from the data during the analysis. All Bayesian analyses were initiated with random starting trees and were run for $10^{7}$ generations. The number of chains was set to four and Markov chains were sampled every 1000 iterations. Points prior to convergence were determined graphically and discarded. Calculated trees were imported into ARB and short sequences obtained in this 
study were subsequently added by use of the ARB parsimony method without changing the tree topology.

\section{Molecular identification of sponge samples}

To verify the identity of the sponge species, the six OTUs that represented the highest number of reads per sponge species (i.e. OTU65: S. massa, OTU190: H. xena, OTU319: A. aerophoba, OTU320: P. ficiformis, OTU333: Axinella damicornis and Axinella verrucosa and OTU495: $H$. panicea) were compared with the non-redundant nucleotide database using the Blastn query (Table S2). Sanger reads larger than 900 nucleotides of the 18S rRNA gene amplicons of $H$. panicea (P1-P3) and $H$. xena $(\mathrm{X} 1-\mathrm{X} 3)$ were published in another study (Naim et al. 2014) with accession numbers KC899022-KC899040. The 18S rRNA gene regions of the amplicons generated here do not overlap with the sequences KC899022-KC899040.

\section{Results}

After DNA sequence quality filtering, a total of 350,341 non-chimeric reads were retained. Thirty five reads could not be classified at the domain level and were removed from the dataset prior to further analysis. The remaining reads clustered into 585 OTUs. In total, 330,884 sequences (107 OTUs), contributing to $94.4 \%$ of all reads, were derived from the phylum Porifera (sponges). When only the sponge samples (and not seawater) were included, the percentage of sequences identified as Porifera increased to $96.8 \%$. $P$. ficiformis was an outlier compared to the other sponge species as more than half of the reads obtained was classified as "non-sponge" (Fig. 1). Fungi represented $13.3 \%$ of the reads (1967 fungal reads) in $P$. ficiformis, and other eukaryotes (defined as eukaryotes that are not sponge and not fungi) comprised $37.8 \%$ of the $18 \mathrm{~S}$ rRNA gene reads. $A$. aerophoba also contained a substantial fraction of non-sponge reads $(7.8 \%)$, but fungi represented only $0.17 \%$ (60 reads) of the $18 \mathrm{~S}$ rRNA gene reads in this sponge species. In the North Sea sponge H. panicea fungi represented $1.5 \%$ (408 reads) of the 18S rRNA gene reads, while nearly no "other eukaryotic" sequences were found. For the other sponge species studied here the large majority of sequences obtained were classified as "sponge", and lower numbers of fungal reads were obtained: 63 for S. massa, 51 for $\mathrm{H}$. xena, 1 for $A$. verrucosa and no fungal reads were found in $A$. damicornis. Generally, the three replicates per species yielded similar numbers of fungal reads (Table 1).

After removal of the 107 Porifera OTUs and 369 OTUs that were classified as "other eukaryotes" from the dataset, the remaining 109 fungal OTUs were used for diversity analyses. Highest fungal richness (Chao1) among the sponge samples was predicted for $P$. ficiformis and $H$. panicea, and richness estimates were generally positively correlated with the number of fungal reads obtained for the sponge samples (Table 1). Although some stratification of the fungal communities can be observed by sponge host species, no obvious discrimination based on geographical regions can be made (Suppl. Fig. 1). 
236 The fungal OTUs detected in sponges belonged to the phyla Ascomycota and Basidiomycota, and four fungal-like OTUs were categorized in the environmental clade LKM11 (Fig. 2 and Fig. 3). OTUs belonging to the phylum Chytridiomycota were only found in seawater.

For a deeper phylogenetic analysis, OTUs obtained as singletons and those that were only found in the seawater samples were disregarded, leaving a final set of 44 fungal OTUs detected in sponges. Twenty-eight of these OTUs were also retrieved from seawater samples. Half of the 44 retained OTUs was identified as yeast (Fig. 4), representing $84 \%$ of the fungal reads found in sponges. The large majority $(79 \%$ of the reads classified as yeasts) of these yeasts was classified to the order Malasseziales. OTU514 was the most dominant fungal OTU detected and belonged to this order. It was detected in all sponge species for which fungal reads were obtained (Fig. 3). Other yeasts encountered in sponges belonged to the orders Saccharomycetales, Leucosporidiales, Tremellales, Cystofilobasidiales, Sporidiobolales, Ustilaginales, Microstromatales and Sporidiales (in order of decreasing relative abundance) (Fig. 3 and 4).

The other 22 fungal OTUs were taxonomically identified as non-yeast fungi and belonged to the orders Eurotiales, Dothidiomycetes, Hypocreales, Chaetothriales, Capnodiales, Heliotales, Polyporales, Ophiostomales, Telochistales, Agaricales, fungal clade LKM11, and a number of OTUs belonging to Ascomycota and Basidiomycota could not be reliably classified at the order level (Fig. 4). The most abundant non-yeast fungal OTU (OTU344) belonged to the order Eurotiales and was closely related to fungi previously isolated from other sponges, but was also present in both Mediterranean and North Sea seawater samples.

Sixteen of the 44 fungal OTUs that were detected in sponges were not detected in seawater. However, only two of these OTUs were represented by more than 10 reads (Fig. 3). These are OTU392, a yeast belonging to the order Saccharomycetales and OTU404, a fungus belonging to the order Chaetothyriales. In addition, several fungal OTUs were found to be present both in seawater and in sponges, but were represented by a higher number of reads in sponges. OTUs that collected substantially more reads in sponges include the most abundant fungal OTU of the dataset, OTU514 (Malasseziales). Other yeasts that collected more reads in sponges than in seawater were OTU183 (order Leucosporidiales, enriched in H. panicea), OTU298 (order Tremellales, enriched in $H$. panicea) and OTU360 (order Saccharomycetales, enriched in H. panicea, H. xena, $S$. massa, $P$. ficiformis, $A$. aerophoba). Furthermore, for the non-yeast fungus OTU344 (order Eurotiales) more reads were obtained from $P$. ficiformis than from seawater. It should be noted, however, that these 'enriched' fungal OTUs in sponges were also all detected in both North Sea and Mediterranean seawater and that they were often found in multiple sponge species (Fig. 3).

\section{Discussion}

The large majority of $18 \mathrm{~S}$ rRNA gene sequences that was obtained from sponges was derived from the sponge hosts. In total 2550 fungal reads were obtained from the sponges 
278 investigated (including the reads obtained as singletons). These reads represented less than $0.75 \%$ of all the reads that were obtained from the sponges. The number of fungal reads obtained from the sponge samples in our study was similar to, or exceeded, the number of fungal reads reported in other studies that have applied next generation sequencing technology to study fungal diversity in sponges (Passarini et al. 2015; Rodríguez-Marconi et al. 2015). Therefore, with respect to numbers, these studies are more comparable to past studies that used clone libraries for diversity estimations of bacterial diversity in sponges (Hentschel et al. 2002; Sipkema \& Blanch 2010) than to studies that apply next generation sequencing technology. That implies that the interpretation and discussion of our results remains mostly limited to qualitative statements as the limited statistical power based on the data did generally not allow quantitative statements. However, one quantitative statement that can be made for the seven sponge studied here is that with the exception of $P$. ficiformis the number of fungal reads was very low in comparison to the number of sponge reads ( 2550 reads vs. 330,884 reads). This is in line with the low relative abundance of fungi in nineteen other sponge species that were assessed by cultivation-independent methods (Gao et al. 2008; He et al. 2014; Passarini et al. 2015; Rodríguez-Marconi et al. 2015; Wang et al. 2014). The low number of fungal reads also corraborates the lack of microscopic observation of fungi reported from sponge tissues. These low numbers and lack of observations are in sharp contrast to the numbers of bacteria that are present in especially high-microbialabundance sponges, and which may account for up to $38 \%$ of the sponge tissue volume (Vacelet \& Donadey 1977).

When analysing the fungal OTUs associated with the sponges, many OTUs were found across different sponge species, across sample types (sponges and seawater) and across geographical regions (Fig. 3). This fungal diversity pattern opposes the bacterial diversity patterns that have been obtained for these sponge species in which every sponge species was observed to have a large fraction of sponge species-specific bacteria, i.e. bacteria found in only one of the sponge species and not or barely found in seawater (Naim et al. 2014; Schmitt et al. 2012; Sipkema et al. 2015). The dominant fungal OTU encountered (OTU514) was a yeast belonging to the order Malasseziales, and it was found in all sponges from which fungal reads were obtained. The Malasseziales were also found to be the dominant order in cultivation-independent fungal diversity studies of the sponges Dragmacidon reticulatum (Passarini et al. 2015), Suberites zeteki and Mycale armata (Gao et al. 2008). It is interesting to note that the nearest neighbours of OTU514 all refer to sequences that have been obtained by cultivation-independent methods (Fig. 4B and Table S3). This observation implies that the dominant fungi observed in sponges are different from the ones obtained through cultivation. That suggests that the 'great plate anomaly' that is known to exist for bacteria in sponges also may be true for fungi found in sponges (Schippers et al. 2012; Sipkema et al. 2011). However, the fact that these yeasts are found in many different sponge species do not yet qualify them as 'sponge-specific' or 'sponge-enriched' fungi as revisited by Simister et al. (2012) as the same or highly similar sequences have been obtained from seawater 
samples in this study and seawater samples from other studies (Fig. 4B and Table S3). Therefore, it is likely that Malasseziales are found in sponges because they are filtered from the seawater rather then that they are in a symbiotic relationship with the sponge host.

Without discussing all OTUs that were found in sponges individually, it can be said that 28 of the 44 fungal OTUs that were observed in sponges were also detected in corresponding seawater samples and these OTUs represented $97.7 \%$ of the fungal reads found in sponges (Fig. 3). The two OTUs that represented $>10$ reads in sponges and were not found in our seawater samples (OTU392 and OTU404) were found most closely related to fungal $18 \mathrm{~S}$ rRNA gene sequences obtained from anaerobic sludge or rockinhabiting fungi (Fig. 4B and Table S3). Therefore, it appears that most -if not all- fungal OTUs obtained in this study are not specifically associated to sponges and that they cannot be classified as 'sponge-specific' or 'sponge-enriched' according to the pioneering review by Simister et al. (2012). Three sponge-specific clusters of fungi were proposed by the latter authors (these clusters are indicated in Fig. 4). A remarkable aspect of the fungi in these proposed clusters is that they are all derived from fungal isolates and not from sequences obtained by cultivation-independent means. This is in sharp contrast to their bacterial and archaeal counterparts, as sponge-specific clusters in the domains Bacteria and Archaea were nearly all obtained from clone libraries (see Supplementary figures in Simister et al. (2012). The state of the art is that we currently know very little about fungi from sponges. Based on the data shown here and other cultivationindependent studies targeting sponge-associated fungi (Gao et al. 2008; He et al. 2014; Passarini et al. 2015; Rodríguez-Marconi et al. 2015) there are indications that we know very little because there is little to be known. The combination of low numbers of fungal reads retrieved from sponges with their unspecific nature based on the currently available data point towards merely accidental presence of fungi in sponges. On the other hand there are a few examples that would advocate a more symbiotic relationship between sponges and fungi. The first record is an unidentified encrusting sponge that grows on top of fungi belonging to the genus Koralionastes (Kohlmeyer \& Volkmann-Kohlmeyer 1990). The second lead stems from the microscopic observations of yeasts that are maternally transmitted in three sponge species belonging to the genus Chondrilla (Maldonado et al. 2005). A better understanding of potential specific sponge-fungi relationships would benefit from further exploring these two known relationships. Also for other marine invertebrates, such as corals and tunicates the nature and consistency of the relationship between the fungi encountered and their hosts have remained unresolved(Yarden 2014). The still ongoing (meta)genomics revolution may be key to shed light on these aspects(Scazzocchio 2014).

\section{Acknowledgements}


We would like to acknowledge Dr. Klaske Schippers and Prof. René Wijfels for sample collection and initial identification of the North Sea sponges and Prof. Maria Uriz for her help with the initial identification of the Mediterranean sponges.

\section{References}

Amend AS, Barshis DJ, and Oliver TA. 2012. Coral-associated marine fungi form novel lineages and heterogeneous assemblages. ISME J6:1291-1301.

Baker PW, Kennedy J, Dobson ADW, and Marchesi JR. 2009. Phylogenetic diversity and antimicrobial activities of fungi associated with Haliclona simulans isolated from Irish coastal waters. Mar Biotechnol 11:540-547.

Bass D, Howe A, Brown N, Barton H, Demidova M, Michelle H, Li L, Sanders H, Watkinson SC, Willcock $S$, and Richards TA. 2007. Yeast forms dominate fungal diversity in the deep oceans. Proc $R$ Soc $B$ 274:3069-3077.

Bentis CJ, Kaufman L, and Golubic S. 2000. Endolithic fungi in reef-building corals (Order: Scleractinia) are common, cosmopolitan, and potentially pathogenic. Biol Bull 198:254-260.

Borchiellini C, Boury-Esnault N, Vacelet J, and Le Parco Y. 1998. Phylogenetic analysis of the Hsp70 sequences reveals the monophyly of Metazoa and specific phylogenetic relationships between animals and fungi. Mol Biol Evol 15:647-655.

Caporaso JG, Bittinger K, Bushman FD, DeSantis TZ, Andersen GL, and Knight R. 2010a. PyNAST: a flexible tool for aligning sequences to a template alignment. Bioinformatics 26:266-267.

Caporaso JG, Kuczynski J, Stombaugh J, Bittinger K, Bushman FD, Costello EK, Fierer N, Peña AG, Goodrich JK, Gordon JI, Huttley GA, Kelley ST, Knights D, Koenig JE, Ley RE, Lozupone CA, McDonald D, Muegge BD, Pirrung M, Reeder J, Sevinsky JR, Turnbaugh PJ, Walters WA, Widmann J, Yatsunenko T, Zaneveld J, and Knight R. 2010b. QIIME allows analysis of high-throughput community sequencing data. Nat Meth 7:335-336.

Castresana J. 2000. Selection of Conserved Blocks from Multiple Alignments for Their Use in Phylogenetic Analysis. Mol Biol Evol17:540-552.

Chao A, and Shen T-J. 2013. User's guide for program SPADE (Species Prediction And Diversity Estimation). 
Chen SC, Chen YC, Manopo I, Wang PC, Chaung HC, Liaw LL, and Chiu SH. 2007. Metschnikowia bicuspidata dominates in Taiwanese cold-weather yeast infections of Macrobrachium rosenbergii. Dis Aquat Organ 75:191-199.

Edgar RC. 2010. Search and clustering orders of magnitude faster than BLAST. Bioinformatics 26:24602461.

Ein-Gil N, Ilan M, Carmeli S, Smith GW, Pawlik JR, and Yarden O. 2009. Presence of Aspergillus sydowii, a pathogen of gorgonian sea fans in the marine sponge Spongia obscura. 3:752-755.

Gao Z, Li B, Zheng C, and Wang G. 2008. Molecular detection of fungal communities in the Hawaiian marine sponges Suberites zetekiand Mycale armata. App/ Environ Microbio/74:6091-6101.

Hamady M, Walker JJ, Harris JK, Gold NJ, and Knight R. 2008. Error-correcting barcoded primers for pyrosequencing hundreds of samples in multiplex. Nat Meth 5:235-237.

Hawksworth DL. 1991. The fungal dimension of biodiversity: magnitude, significance, and conservation. Mycol Res 95:641-655.

Hawksworth DL. 2001. The magnitude of fungal diversity: the 1.5 million species estimate revisited. Mycol Res 105:1422-1432.

He L, Liu F, Karuppiah V, Ren Y, and Li Z. 2014. Comparisons of the fungal and protistan communities among different marine sponge holobionts by pyrosequencing. Microb Eco/67:951-961.

Hentschel U, Hopke J, Horn M, Friedrich AB, Wagner M, Hacker J, and Moore BS. 2002. Molecular evidence for a uniform microbial community in sponges from different oceans. Appl Environ Microbiol 68:4431-4440.

Höller U, Wright AD, Matthée GF, Konig GM, Draeger S, Aust HJ, and Schulz B. 2000. Fungi from marine sponges: diversity biological activity and secondary metabolites. Mycol Res 104:1354-1365.

Hyde KD, Jones EBG, Leaño E, Pointing SB, Poonyth AD, and Vrijmoed LLP. 1998. Role of fungi in marine ecosystems. Biodiver Conserv 7:1147-1161.

Indraningrat A, Smidt $H$, and Sipkema D. 2016. Bioprospecting sponge-associated microbes for antimicrobial compounds. Mar Drugs 14:87. 
Jin L, Liu F, Sun W, Zhang F, Karuppiah V, and Li Z. 2014. Pezizomycotina dominates the fungal communities of South China Sea Sponges Theonella swinhoei and Xestospongia testudinaria. FEMS Microbiol Ecol 90:935-945.

Kis-Papo T, Oren A, Wasser SP, and Nevo E. 2003. Survival of filamentous fungi in hypersaline Dead Sea water. Microb Ecol45:183-190.

Kohlmeyer J, and Volkmann-Kohlmeyer B. 1990. New species of Koralionastes (Ascomycotina) from the Caribbean and Australia. Can J Bot 68:1554-1559.

Lara E, Moreira D, and López-García P. 2010. The environmental clade LKM11 and Rozella form the deepest branching clade of Fungi. Protist 161:116-121.

Le Calvez T, Burgaud G, Mahé S, Barbier G, and Vandenkoornhuyse P. 2009. Fungal diversity in deepsea hydrothermal ecosystems. App/ Environ Microbio/ 75:6415-6421.

Liu WC, Li CQ, Zhu P, Yang JL, and Cheng KD. 2010. Phylogenetic diversity of culturable fungi associated with two marine sponges: Haliclona simulans and Gelliodes carnosa, collected from the Hainan Island coastal waters of the South China Sea. Fungal Divers 42:1-15.

Loque CP, Medeiros AO, Pellizzari FM, Oliveira EC, Rosa CA, and Rosa LH. 2009. Fungal community associated with marine macroalgae from Antarctica. Polar Bio/33:641-648.

Ludwig W, Strunk O, Westram R, Richter L, Meier H, Yadhukumar, Buchner A, Lai T, Steppi S, Jobb G, Förster W, Brettske I, Gerber S, Ginhart AW, Gross O, Grumann S, Hermann S, Jost R, König A, Liss T, Lüßmann R, May M, Nonhoff B, Reichel B, Strehlow R, Stamatakis A, Stuckmann N, Vilbig A, Lenke M, Ludwig T, Bode A, and Schleifer KH. 2004. ARB: a software environment for sequence data. Nucleic Acids Res 32:1363-1371.

Maldonado M, Cortadellas N, Trillas MI, and Rützler K. 2005. Endosymbiotic yeast maternally transmitted in a marine sponge. Biol Bull 209.

Mueller GM, and Schmit JP. 2007. Fungal biodiversity: what do we know? What can we predict? Biodivers Conserv 16:1-5.

Naim MA, Morillo JA, Sørensen SJ, Waleed AA-S, Smidt H, and Sipkema D. 2014. Host-specific microbial communities in three sympatric North Sea sponges. FEMS Microbiol Ecol 90:390-403.

Passarini MR, Santos C, Lima M, Berlinck RG, and Sette LD. 2013. Filamentous fungi from the Atlantic marine sponge Dragmacidon reticulatum. Arch Microbiol 195:99-111. 
447 Passarini MRZ, Miqueletto PB, de Oliveira VM, and Sette LD. 2015. Molecular diversity of fungal and 448 bacterial communities in the marine sponge Dragmacidon reticulatum. Journal of Basic Microbiology $449 \quad 55: 207-220$

450

451

452

453

Paz Z, Komon-Zelazowska M, Druzhinina IS, Aveskamp MM, Shnaiderman A, Aluma Y, Carmeli S, Ilan M, and Yarden O. 2010. Diversity and potential antifungal properties of fungi associated with a Mediterranean sponge. Fungal Divers 42:17-26.

Pivkin MV, Aleshko SA, Krasokhin VB, and Khudyakova YV. Fungal assemblages associated with sponges of the southern coast of Sakhalin Island. Russ J Mar Bio/32:207-213.

Pruesse E, Quast C, Knittel K, Fuchs BM, Ludwig W, Peplies J, and Glöckner FO. 2007. SILVA: a comprehensive online resource for quality checked and aligned ribosomal RNA sequence data compatible with ARB. Nucl Acids Res 35:7188-7196.

Richards TA, Jones MDM, Leonard G, and Bass D. 2012a. Marine fungi: their ecology and molecular diversity. Ann Rev Mar Sci 4:495-522.

Richards TA, Jones MDM, Leonard G, and Bass D. 2012b. Marine fungi: their ecology and molecular diversity. Annu Rev Mar Sci 4:495-522.

Richards TA, Leonard G, Mahé F, del Campo J, Romac S, Jones MDM, Maguire F, Dunthorn M, De Vargas C, Massana R, and Chambouvet A. 2015. Molecular diversity and distribution of marine fungi across 130 European environmental samples. Proc R Soc B 282:20152243.

Rodríguez-Marconi S, De la Iglesia R, Díez B, Fonseca CA, Hajdu E, and Trefault N. 2015. Characterization of bacterial, archaeal and eukaryote symbionts from Antarctic sponges reveals a high diversity at a three-domain level and a particular signature for this ecosystem. PLOS ONE 10:e0138837.

Rodriguez RJ, Redman RS, and Henson JM. 2004. The role of fungal symbioses in the adaptation of plants to high stress environments. Mitig Adapt Strat Global Change 9:261-272.

Ronquist F, Teslenko M, van der Mark P, Ayres DL, Darling A, Höhna S, Larget B, Liu L, Suchard MA, and Huelsenbeck JP. 2012. MrBayes 3.2: efficient Bayesian phylogenetic inference and model choice across a large model space. Syst Bio/61:539-542.

Rot C, Goldfarb I, Ilan M, and Huchon D. 2006. Putative cross-kingdom horizontal gene transfer in sponge (Porifera) mitochondria. BMC Evol Bio/6:1-11.

Scazzocchio C. 2014. Fungal biology in the post-genomic era. Fungal Biol Biotechnol 1:7. 
Schippers KJ, Sipkema D, Osinga R, Smidt H, Pomponi SA, Martens DE, and Wijffels RH. 2012. Cultivation of sponges, sponge cells and symbionts: achievements and future prospects. Adv Mar Bio/62:273-337.

Schmitt S, Hentschel U, and Taylor MW. 2012. Deep sequencing reveals diversity and community structure of complex microbiota in five Mediterranean sponges. Hydrobiologia 687:341-351.

Simister RL, Deines P, Botté ES, Webster NS, and Taylor MW. 2012. Sponge-specific clusters revisited: a comprehensive phylogeny of sponge-associated microorganisms. Environ Microbio/ 14:517-524.

Singh P, Raghukumar C, Verma P, and Shouche Y. 2010. Fungal community analysis in the deep-sea sediments of the Central Indian Basin by culture-independent approach. Microb Eco/61:507-517.

Sipkema D, and Blanch HW. 2010. Spatial distribution of bacteria associated with the marine sponge Tethya californiana. Mar Biol 157:627-638.

Sipkema D, de Caralt S, Morillo JA, Al-Soud WA, Sørensen SJ, Smidt H, and Uriz MJ. 2015. Similar spongeassociated bacteria can be acquired via both vertical and horizontal transmission. Environ Microbiol 17:3807-3821.

Sipkema D, Schippers K, Maalcke WJ, Yang Y, Salim S, and Blanch HW. 2011. Multiple approaches to enhance the cultivability of bacteria associated with the marine sponge Haliclona (Gellius) sp. App/ Environ Microbio/77:2130-2140.

Taylor MW, Radax R, Steger D, and Wagner M. 2007. Sponge-associated microorganisms: evolution, ecology and biotechnological potential. Microbiol Mol Biol Rev 71:295-347.

Toledo-Hernández C, Zuluaga-Montero A, Bones-González A, Rodríguez JA, Sabat AM, and Bayman P. 2008. Fungi in healthy and diseased sea fans (Gorgonia ventalina): is Aspergillus sydowii always the pathogen? Coral Reefs 27:707-714.

Vaca I, FaロndezC, Maza F, Paillavil B, Hernández V, Acosta F, Levicán G, Martínez C, and Chávez R. 2013. Cultivable psychrotolerant yeasts associated with Antarctic marine sponges. World J Microbiol Biotechnol 29:183-189.

Vacelet J, and Donadey C. 1977. Electron microscope study of the association between some sponges and bacteria. J Exp Mar Biol Eco/30:301-314.

Vainio EJ, and Hantula J. 2000. Direct analysis of wood-inhabiting fungi using denaturing gradient gel electrophoresis of amplified ribosomal DNA. Mycol Res 104:927-936. 
504 Wang G, Li Q, and Zhu P. 2007. Phylogenetic diversity of culturable fungi associated with the Hawaiian 505 Sponges Suberites zeteki and Gelliodes fibrosa. Antonie van Leeuwenhoek 93:163-174.

506

507

508

509

510

511

512

513

Wang Y, Tian RM, Gao ZM, Bougouffa S, and Qian P-Y. 2014. Optimal Eukaryotic 18S and universal 16S/18S ribosomal RNA primers and their application in a study of symbiosis. PLOS ONE 9:e90053.

Webster NS, and Taylor MW. 2012. Marine sponges and their microbial symbionts: love and other relationships. Environ Microbio/14:335-346.

Wiese J, Ohlendorf B, Blümel M, Schmaljohann R, and Imhoff JF. 2011. Phylogenetic identification of fungi isolated from the marine sponge Tethya aurantium and identification of their secondary metabolites. Mar Drugs 9:561-585.

Yarden O. 2014. Fungal association with sessile marine invertebrates. Front Microbio/5. 
514

515

516

517

518

519

520

521

522

523

524

525

526

527

528

529

530

531

532

533

534

535

536

537

538

539

540

541

542

543

544

545

546

547

548

549

550

551

552

553

554

555

\section{Figure Legends}

Figure 1. Relative abundance of sponge, fungal and "other eukaryotic" $18 \mathrm{~S}$ rRNA gene sequences for sample types from the North Sea (A-D) and Mediterranean Sea (E-I). The triplicate samples per sponge species are pooled and the numbers within the pie diagrams represent the number of sequences obtained for the sample type. The numbers in parentheses indicate the number of fungal reads obtained for each sample type. WNS = North Sea water; WMS = Mediterranean seawater.

Figure 2. Relative abundance of fungal phyla found in the cumulative sponge and seawater samples.

Figure 3. Heatmap of fungal OTUs in North Sea sponges and Mediterranean Sea sponges. OTUs that were only found in seawater and singletons are not shown. Taxonomic affiliation is shown at phylum level and order level whenever possible. Some OTUs (in red) were re-classified based on Bayesian phylogenetic analysis (see also Figure 4). WNS = North Sea water; $\mathrm{P}=$ Halichondria panicea; $\mathrm{X}=$ Haliclona xena; $\mathrm{M}=$ Suberites massa; WMS = Mediterranean seawater; $\mathrm{F}=$ Petrosia ficiformis; $\mathrm{A}=$ Aplysina aerophoba. Numbers 1, 2 and 3 refer to different individuals of the sponge species.

Figure 4. Bayesian phylogram of Ascomycota (A) and Basidiomycota (B) of 44 fungal OTUs found in sponges based on 18S rRNA gene sequences. Other sequences included are i) their nearest neighbors, and ii) 18S rRNA sequences published by (Simister et al. 2012) and (Baker et al. 2009). Numbers in parentheses after the sample type indication refer to the absolute numbers of reads that were included in the corresponding OTU per sample (34 P(0/0/4) WMS(2) $\mathrm{F}(4 / 0 / 0)$ means that 4 reads of $\mathrm{P} 3,2$ reads of Mediterranean seawater and 4 reads of $\mathrm{F} 1$ are included in OTU34). The NCBI genbank accession number is the last descriptor for each branch. Grey boxes indicate sponge-specific clusters (SSC) as defined by Simister (Simister et al. 2012). Yellow boxes represent yeasts. Taxonomic groups marked with an asterisk $\left(^{*}\right)$ indicate non-formal taxonomic classification (i.e. fungal environmental clade LKM11 is not a phylum, but a group assigned for environmental fungal-like sequences (Lara et al. 2010). The numbers above or below the branches correspond to posterior probability (PP) values of the Bayesian analysis. Nodes with PP values of $<50$ are not indicated.

\section{Table Legends}

Table 1. Number of reads, number of fungal reads, observed fungal OTUs, expected fungal OTUs (Chao1) and Coverage (Good's coverage) in seawater and sponge samples at a $97 \%$ sequence similarity threshold.

\section{Supplementary Figure Legends}

Figure S1. Principal coordinate analysis (PCoA) ordinations of fungal communities in the different sponge hosts and seawaters. Only samples with more than 10 fungal reads were included. Plots are based on square root transformed relative abundance data of OTUs $(A)$ or presence-absence data $(B)$. The percentage of total variation that is explained by each PCoA axis is given in the parentheses. 
556

557

558

559

560

561

562

563

564

565

566

567

\section{Supplementary Table Legends}

Table S1. List of barcode sequences and ERS accession numbers used in this study. Sample codes indicate $H$. panicea (P1-P3), H. xena (X1-X3), S. massa (M1-M3), P. ficiformis (F1-F3), A. aerophoba (A1-A3), A. damicornis (D1-D3), A. verrucosa (V1-V3) and sea water (WNS \& WMS).

Table S2. Blastn query at 11-Jan-2017 of selected representative OTUs with highest number of sponge (Porifera) reads from pyrosequencing data from each of the sponge species (and also each of the individual samples belonging to that species) against the non-redundant nucleotide database.

Table S3. BLASTn of OTUs 514, 392 and 404 representative sequence against the NCBI nr/nt database 


\section{Figure 1}

Relative abundance of sponge, fungal and "other eukaryotic" 18S rRNA gene sequences for sample types from the North Sea (A-D) and Mediterranean Sea (E-I)

The triplicate samples per sponge species are pooled and the numbers within the pie diagrams represent the number of sequences obtained for the sample type. The numbers in parentheses indicate the number of fungal reads obtained for each sample type. WNS = North Sea water; WMS = Mediterranean seawater.

A) WNS

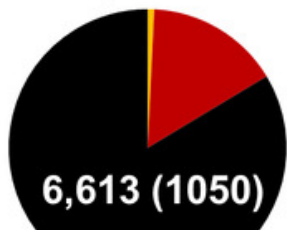

B) H. panicea

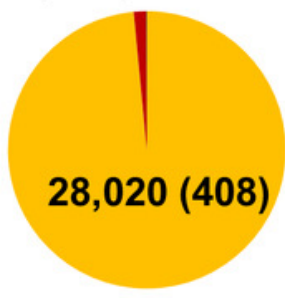

E) WMS

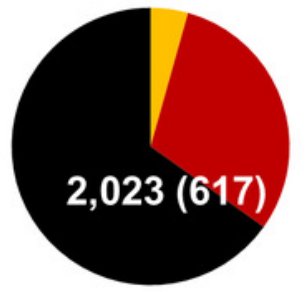

F) $P$. ficiformis
C) H. xena
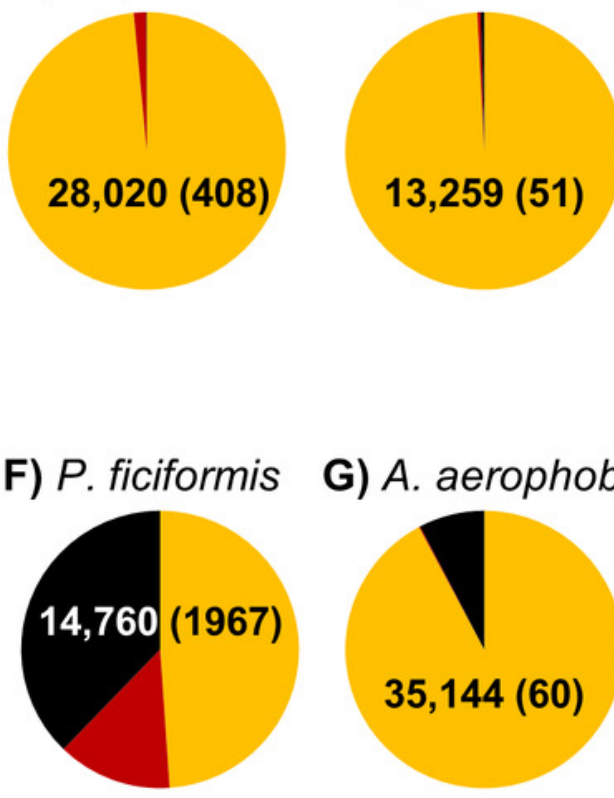

G) A. aerophoba

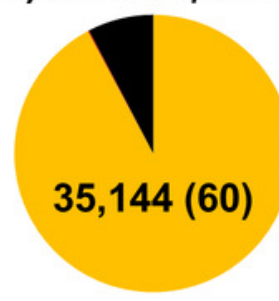

D) S. massa

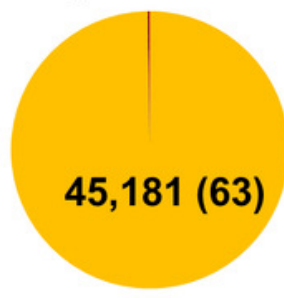

sponge

- fungus

- other eukaryotes 


\section{Figure 2}

Relative abundance of fungal phyla found in the cumulative sponge and seawater samples.
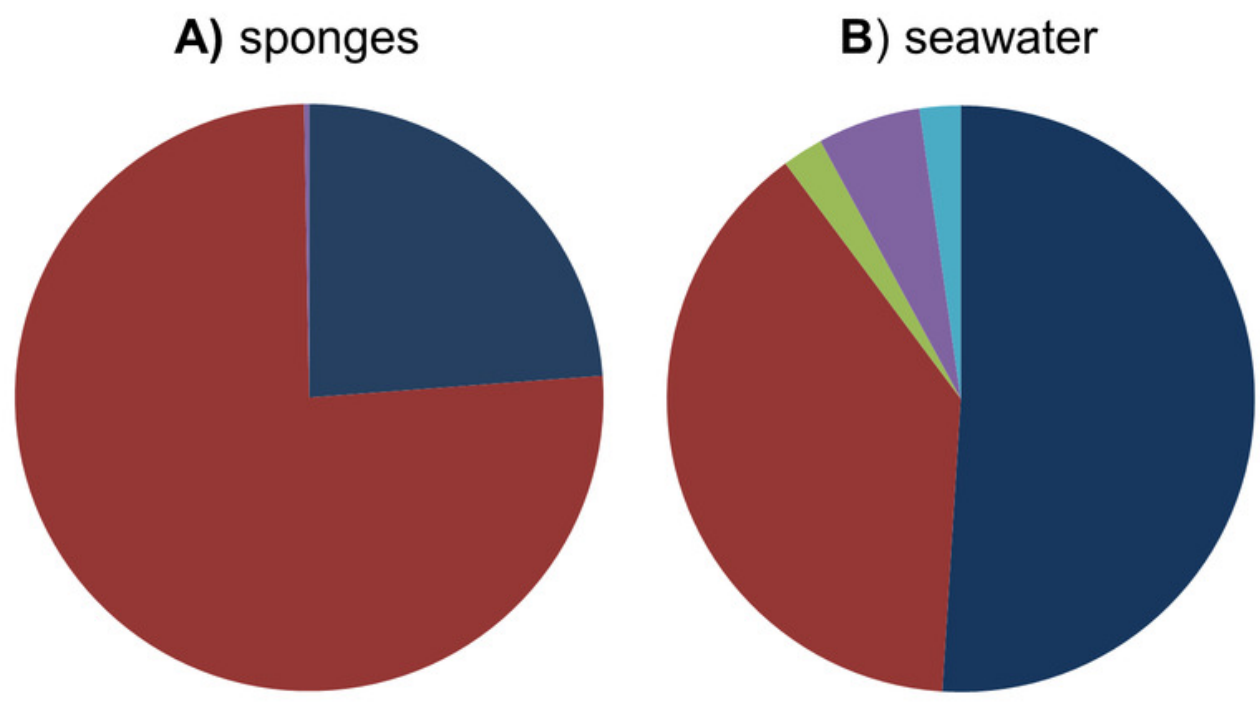

Ascomycota

- Basidiomycota

Chytridiomycota

Environmental clade LKM11

Fungi, unclassified 


\section{Figure 3}

Heatmap of fungal OTUs in North Sea sponges and Mediterranean Sea sponges.

OTUs that were only found in seawater and singletons are not shown. Taxonomic affiliation is shown at phylum level and order level whenever possible. Some OTUs (in red) were reclassified based on Bayesian phylogenetic analysis (see also Figure 4). WNS $=$ North Sea water; $\mathrm{P}=$ Halichondria panicea; $\mathrm{X}=$ Haliclona xena; $\mathrm{M}=$ Suberites massa; $\mathrm{WMS}=$ Mediterranean seawater; $F=$ Petrosia ficiformis; $A=$ Aplysina aerophoba. Numbers 1, 2 and 3 refer to different individuals of the sponge species. 
\#OTU ID Phylum; Order

458 Ascomycota; Capnodiales 265 Ascomycota; Capnodiales 404 Ascomycota; Chaetothyriales

1 Ascomycota; Chaetothyriales 77 Ascomycota; Dothidiomycetes 350 Ascomycota; Dothidiomycetes 509 Ascomycota; Dothidiomycetes 344 Ascomycota; Eurotiales 242 Ascomycota; Heliotales 328 Ascomycota; Heliotales 230 Ascomycota; Hypocreales 546 Ascomycota; Hypocreales 119 Ascomycota; Ophiostomatales 168 Ascomycota; Saccharomycetales 182 Ascomycota; Saccharomycetales 360 Ascomycota; Saccharomycetales 545 Ascomycota; Saccharomycetales 392 Ascomycota; Saccharomycetales 263 Ascomycota; Saccharomycetales 170 Ascomycota; Saccharomycetales 424 Ascomycota;Telochistales 196 Basidiomycota; Agaricales 132 Basidiomycota; Corticiales 232 Basidiomycota; Cystofilobasidiales 183 Basidiomycota; Leucosporidiales 201 Basidiomycota; Malasseziales 341 Basidiomycota; Malasseziales 514 Basidiomycota; Malasseziales 28 Basidiomycota; Malasseziales 479 Basidiomycota; Malasseziales 552 Basidiomycota; Microstromatales 406 Basidiomycota; Polyporales 218 Basidiomycota; Polyporales 19 Basidiomycota; Sporidiobolales 227 Basidiomycota; Sporidiobolales 553 Basidiomycota; Tremellales 298 Basidiomycota; Tremellales 332 Basidiomycota; Tremellales 383 Basidiomycota; Tremellales 499 Basidiomycota; Ustilaginales 206 Environmental clade LKM11 27 Environmental clade LKM11 12 Environmental clade LKM11 67 Environmental clade LKM11

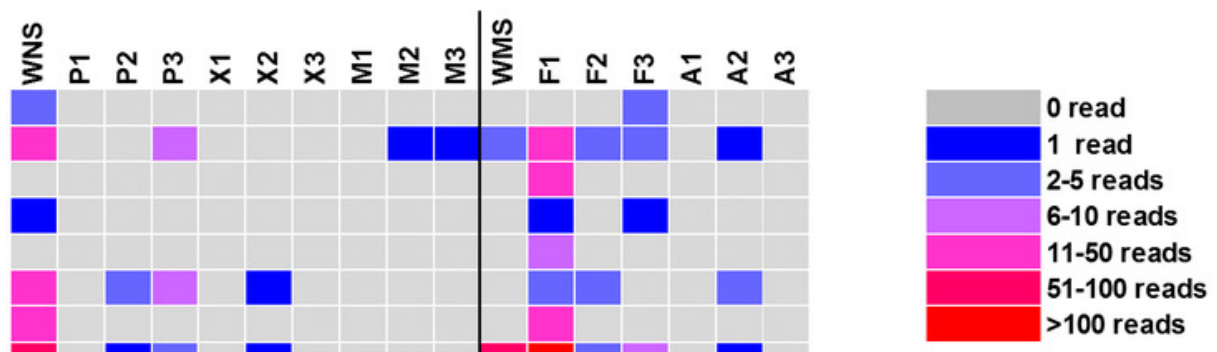




\section{Figure 4}

Bayesian phylogram of Ascomycota (A) and Basidiomycota (B) of 44 fungal OTUs found in sponges based on $18 \mathrm{~S}$ rRNA gene sequences.

Other sequences included are i) their nearest neighbors, and ii) $18 \mathrm{~S}$ rRNA sequences published by ( Simister et al. 2012 ) and (Baker et al. 2009 ). Numbers in parentheses after the sample type indication refer to the absolute numbers of reads that were included in the corresponding OTU per sample (34 P(0/0/4) WMS(2) F(4/0/0) means that 4 reads of $\mathrm{P} 3,2$ reads of Mediterranean seawater and 4 reads of F1 are included in OTU34). The NCBI genbank accession number is the last descriptor for each branch. Grey boxes indicate sponge-specific clusters (SSC) as defined by Simister ( Simister et al. 2012 ). Yellow boxes represent yeasts. Taxonomic groups marked with an asterisk (*) indicate non-formal taxonomic classification (i.e. fungal environmental clade LKM11 is not a phylum, but a group assigned for environmental fungal-like sequences ( Lara et al. 2010 ) . The numbers above or below the branches correspond to posterior probability (PP) values of the Bayesian analysis. Nodes with PP values of $<50$ are not indicated. 


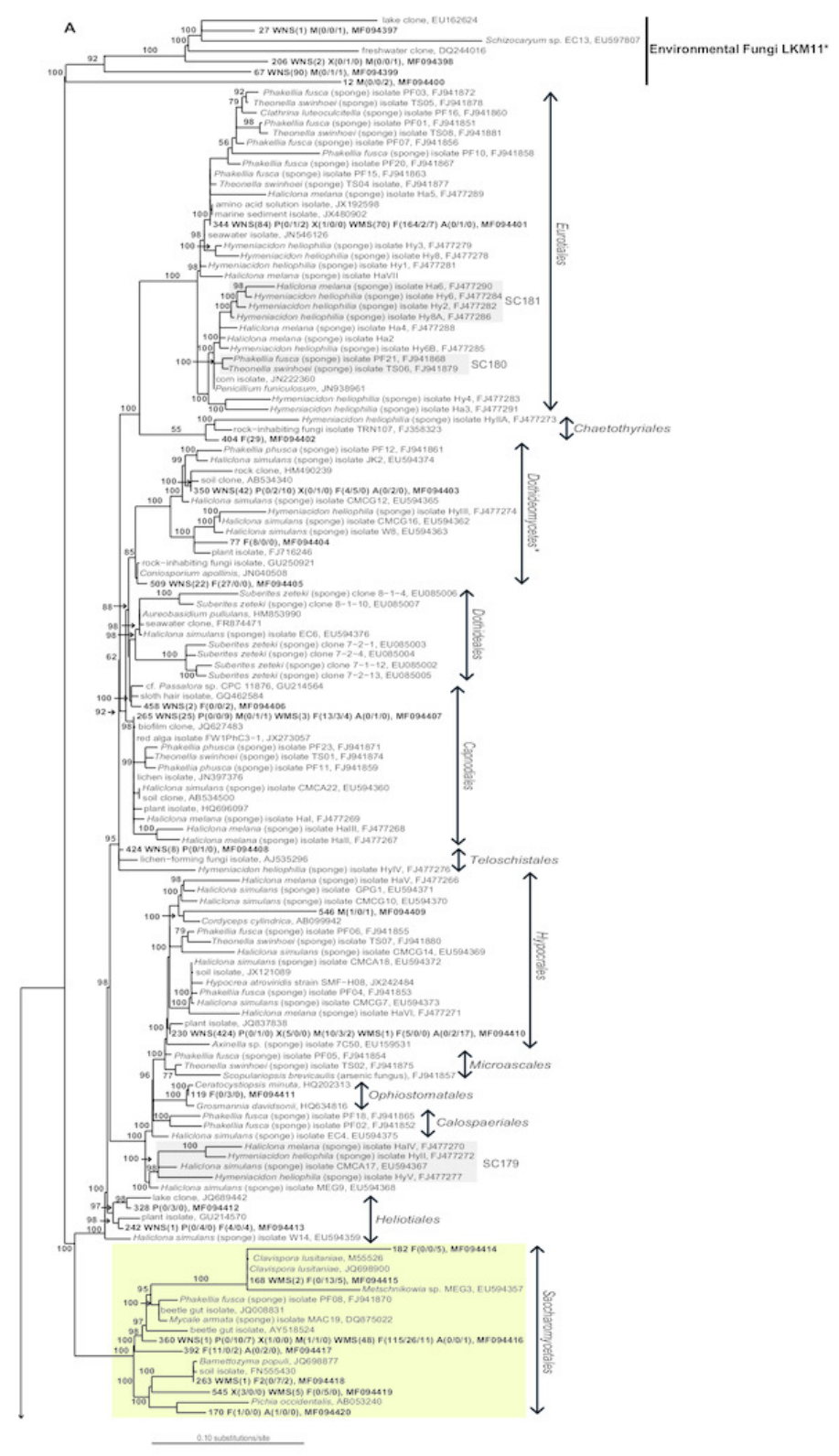

B

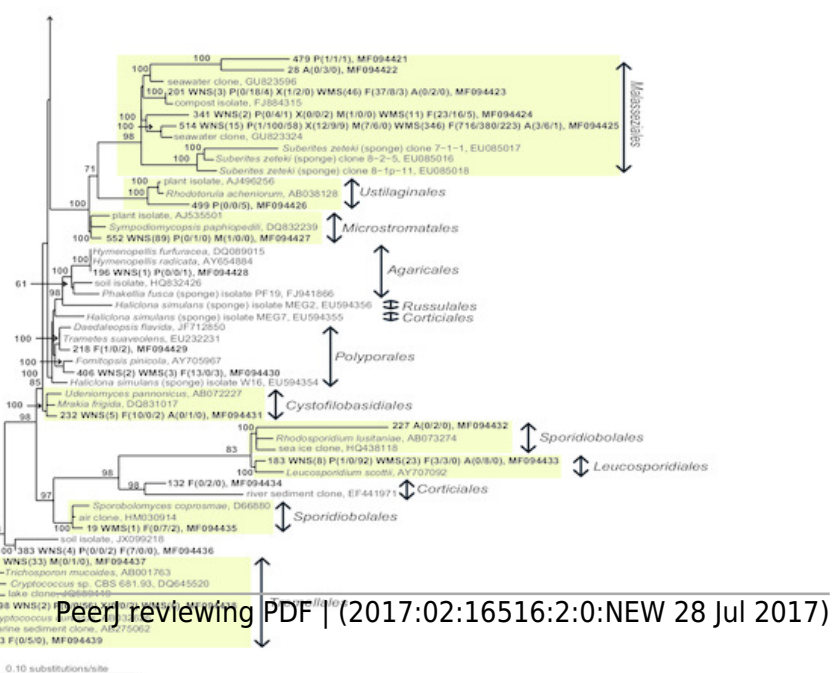




\section{Table $\mathbf{1}$ (on next page)}

Number of reads, number of fungal reads, observed fungal OTUs, expected fungal OTUs (Chaol) and Coverage (Good's coverage) in seawater and sponge samples at a $97 \%$ sequence similarity threshold. 


\begin{tabular}{|c|c|c|c|c|c|c|c|}
\hline \multirow{2}{*}{ Sample Name } & \multirow{2}{*}{ Abbrev. } & \multirow{2}{*}{$\begin{array}{l}\text { Sponge taxonomic } \\
\text { order }\end{array}$} & \multirow{2}{*}{$\begin{array}{l}\text { Total no. of filtered } \\
\text { reads }\end{array}$} & \multirow{2}{*}{ No. of fungal reads } & \multicolumn{3}{|c|}{ fungal reads } \\
\hline & & & & & Observed OTUs & Expected OTUs & Coverage \\
\hline North Sea water & WNS & & 6613 & 1050 & 69 & $94 \pm 13$ & 0.97 \\
\hline H. panicea 1 & P1 & Halichondrida & 12418 & 3 & 3 & $6 \pm 4$ & 0 \\
\hline H. panicea 2 & P2 & Halichondrida & 8389 & 149 & 15 & $29 \pm 13$ & 0.95 \\
\hline H. panicea 3 & P3 & Halichondrida & 7214 & 256 & 16 & $18 \pm 3$ & 0.98 \\
\hline H. xena 1 & $\mathbf{X} 1$ & Haplosclerida & 3728 & 22 & 5 & $6 \pm 2$ & 0.91 \\
\hline H. xena 2 & X2 & Haplosclerida & 4155 & 14 & 5 & $7 \pm 3$ & 0.79 \\
\hline H. xena 3 & $\mathbf{X 3}$ & Haplosclerida & 5381 & 15 & 5 & $5 \pm 1$ & 0.87 \\
\hline S. massa 1 & M1 & Hadromerida & 14265 & 25 & 8 & $18 \pm 10$ & 0.8 \\
\hline S. massa 2 & M2 & Hadromerida & 19955 & 27 & 9 & $17 \pm 8$ & 0.78 \\
\hline S. massa 3 & M3 & Hadromerida & 10961 & 11 & 9 & $16 \pm 7$ & 0.36 \\
\hline Med. Sea water & WMS & & 2023 & 617 & 17 & $17 \pm 1$ & 1.00 \\
\hline P. ficiformis 1 & F1 & Haplosclerida & 5704 & 1200 & 23 & $29 \pm 7$ & 1.00 \\
\hline P. ficiformis 2 & F2 & Haplosclerida & 4623 & 486 & 17 & $17 \pm 0$ & 1.00 \\
\hline P. ficiformis 3 & F3 & Haplosclerida & 4462 & 281 & 16 & $16 \pm 0$ & 1.00 \\
\hline A. aerophoba 1 & A1 & Verongida & 14900 & 7 & 4 & $5 \pm 1$ & 0.71 \\
\hline A. aerophoba 2 & A2 & Verongida & 15285 & 32 & 13 & $15 \pm 2$ & 0.84 \\
\hline A. aerophoba 3 & $\mathbf{A 3}$ & Verongida & 17472 & 21 & 21 & $11 \pm 4$ & 0.81 \\
\hline A. damicornis 1 & D1 & Halichondrida & 14900 & 0 & 0 & - & - \\
\hline A. damicornis 2 & D2 & Halichondrida & 15572 & 0 & 0 & - & - \\
\hline A. damicornis 3 & D3 & Halichondrida & 17472 & 0 & 0 & - & - \\
\hline A. verrucosa 1 & V1 & Halichondrida & 13583 & 0 & 0 & - & - \\
\hline A. verrucosa 2 & $\mathbf{V} 2$ & Halichondrida & 13701 & 0 & 0 & - & - \\
\hline A. verrucosa 3 & V3 & Halichondrida & 130365 & 1 & 1 & $1 \pm 0$ & 0 \\
\hline
\end{tabular}

